\title{
Psicologia Positiva: uma nova abordagem para antigas questões
}

\author{
Simone dos Santos Paludo \\ Sílvia Helena Koller \\ Universidade Federal do Rio Grande do Sul, Porto Alegre, Brasil
}

\begin{abstract}
Resumo: Historicamente, a Psicologia preocupou-se em investigar patologias, negligenciado os aspectos saudáveis dos seres humanos. Mas, a partir de 1998, assumindo a presidência da American Psychological Association, Seligman iniciou movimento denominado Psicologia Positiva, que visa oferecer nova abordagem às potencialidades e virtudes humanas, estudando as condições e processos que contribuem para a prosperidade dos indivíduos e comunidades. Este artigo apresenta e discute essa nova proposta científica, que promete melhorar a qualidade de vida dos indivíduos e prevenir patologias; também as lacunas existentes nas investigações em Ciências Humanas, as contribuições teóricas e empíricas e as possíveis aplicações desse conhecimento.
\end{abstract}

Palavras-chave: Psicologia positiva. Potencialidades humanas. Histórico da investigação psicológica.

\section{Positive Psychology: a new approach to old questions}

\begin{abstract}
Throughout history, Psychology studies were focused on pathologies, neglecting issues related to human healthy state. In 1998, when Seligman assumed the head of American Psychological Association, he started the Positive Psychology movement, which objective is to offer a new approach to human strengthens and virtues, by studying the conditions and processes that contribute to the prosperity of individuals and communities. The present paper presents and discusses this new scientific approach that intends to promote the quality of life at individual and community levels, preventing pathologies. We also point out its gaps in Human Sciences-directed investigations, as well as its theoretical and empirical contributions and applications.
\end{abstract}

Keywords: Positive psychology. Human potentiality. History of psychological investigation.

\section{Psicología Positiva: un nuevo acercamiento para antiguas cuestiones}

Resumen: Históricamente, la Psicología se preocupó por investigar patologías, descuidándose de los aspectos saludables de los seres humanos. Pero, desde 1998, Cuando Seligman, asumió la presidencia de la American Psychological Association, se inició el movimiento denominado Psicología Positiva, cuyo objetivo fue dar una nueva mirada a las potencialidades y virtudes humanas, estudiando las condiciones y los procesos que contribuyen para la prosperidad de los individuos y comunidades. Este articulo presenta y discute esta nueva propuesta científica, que promete mejorar la calidad de vida de los individuos y prevenir patologías; así como indicar las lagunas producidas en las investigaciones de las Ciencias Humanas, sus contribuciones teóricas, empíricas y posibles aplicaciones.

Palabras clave: Psicología positiva. Potencialidades humanas. Historia de la investigación psicológíca. 
Nos últimos anos, muitas tragédias têm sido anunciadas pelos principais meios de comunicação em todo o mundo - guerras, criminalidade, doenças, pobreza, tráfico de drogas, mortalidade infantil, entre outros. Devido a tal evidência, o destaque a esses eventos parece contraditório aos aspectos positivos da natureza humana. Pergunta-se, então, como a ciência poderia investigar as causas da felicidade se existem, por exemplo, outros fatores mais emergentes, como as causas da depressão? Mas, será que o progresso da ciência deve ser orientado somente pelas emergências? Ou pode se ocupar de outros temas?

Como primeira resposta, tem-se a Psicologia abordando essas questões dentro de um novo movimento científico intitulado Psicologia Positiva, que, nessa nova proposta científica promete melhorar a qualidade de vida dos indivíduos e prevenir as patologias.

O movimento pela Psicologia Positiva teve início em 1998, quando o psicólogo Martin Seligman assumiu a presidência da American Psychological Association (APA). Segundo ele, a ciência psicológica vinha negligenciando o estudo dos aspectos virtuosos da natureza humana, o que pode ser confirmado por uma simples pesquisa no banco de dados da PsycInfo. Ao utilizar a palavra-chave "depressão" são encontrados 110382 artigos entre os anos de 1970 e 2006, por outro lado, a palavra-chave "felicidade" indica apenas 4711 artigos publicados no mesmo período, ou seja, menos da metade.

Para evidenciar a realidade da produção cientifica em Psicologia, Seligman e Czikszentmihalyi publicaram uma edição especial da American Psychologist em janeiro de 2000, na qual enfatizaram que a Psicologia não produzia conhecimento suficiente sobre os aspectos virtuosos e as forças pessoais que todos seres humanos possuem. Nessa importante publicação, apontaram as lacunas presentes nas investigações psicológicas e destacaram a necessidade de pesquisas sobre aspectos positivos como, por exemplo, esperança, criatividade, coragem, sabedoria, espiritualidade, felicidade.

No entanto, em cinco anos, houve grande expansão e muito tem acontecido nesse movimento científico; é grande o número de artigos e livros publicados, conforme mostra a literatura (Aspinwall \& Staudinger, 2003; Compton, 2005; Keyes \& Haidt,
2003; Lopez \& Snyder, 2003; Peterson \& Seligman, 2004; Schmuck \& Sheldon, 2001; Snyder \& Lopez, 2002); são muitas as conferências, cursos, financiamentos e prêmios oferecidos a pesquisadores do mundo inteiro a fim de fortalecer e divulgar o estudo empírico e teórico. A Psicologia Positiva está, pois, em pleno processo de expansão dentro da ciência psicológica, a qual possibilita uma reavaliação das potencialidades e virtudes humanas por meio do estudo das condições e processos que contribuem para a prosperidade.

De acordo com essa nova visão, o conhecimento das forças e virtudes poderia propiciar o "florescimento" (flourishing) das pessoas, comunidades e instituições. E florescimento tem sido um termo bastante utilizado na Psicologia Positiva, sendo definido por Keyes e Haidt (2003), como uma condição que permite o desenvolvimento pleno, saudável e positivo dos aspectos psicológicos, biológicos e sociais dos seres humanos. Keyes e Haidt (2003) salientam, ainda, que o florescimento significa um estado no qual os indivíduos sentem uma emoção positiva pela vida, apresentam um ótimo funcionamento emocional e social e não possuem problemas relacionados à saúde mental, o que não quer dizer ser um "super-homem ou super-mulher", mas indivíduos considerados em pleno florescimento são aqueles que vivem intensamente mais do que meramente existem (Keyes \& Haidt, 2003).

\section{O processo histórico}

A negligência ao estudo dos aspectos positivos e virtuosos dos seres humanos pela Ciência Psicológica, de acordo com Seligman (2002), baseou-se historicamente no pensamento dominante na Psicologia direcionado ao estudo dos aspectos "anormais". Parece que o fator mais intrigante no estudo do comportamento humano não era representado pela média da população, mas o improvável e o diferente. Embora tenham surgido psicólogos humanistas como, Abraham Maslow (1954) e Carl Rogers (1959), comprometidos com uma nova visão e perspectiva sobre o comportamento humano, suas idéias não pareceram ser suficientemente atrativas e, conseqüentemente, não produziram dados empíricos suficientes para dar força a uma visão mais positiva do ser humano. Seligman e Csikszentmihalyi (2000) apontam a falta 
de rigor metodológico e a inconsistência dos resultados como principais responsáveis pelo enfraquecimento da Psicologia Humanista. Talvez esses autores estivessem à frente de seu tempo e, por isso, suas idéias não foram reconhecidas como necessárias à Psicologia, naquele momento.

Existem importantes discussões sobre as interlocuções e as aproximações teóricas e conceituais entre a Psicologia Humanista e a Psicologia Positiva. Hernandez (2003) apresenta as diferentes manifestações sobre a origem das idéias que fundamentam essa nova abordagem psicológica. Os pesquisadores humanistas dividem-se em dois grandes grupos, aqueles que questionam as proposições de Seligman (Resnick, Warmoth \& Serlin, 2001; Taylor, 2001) e aqueles que as aceitam (Pajares, 2001; Sollod, 2000). Certamente o movimento da Psicologia Positiva compartilha e resgata conceitos e objetivos propostos pela terceira força em Psicologia. Hernandez (2003) propõe uma parceria entre as novas idéias do presente e as novas idéias do passado e sugere a necessidade de estudos que explorem essas relações.

É importante reconhecer que a Psicologia Humanista enfatizou aspectos positivos do desenvolvimento humano, no entanto, suas contribuições científicas receberam pouca atenção no passado. Inúmeros motivos podem ser apontados para justificar a preferência pelos aspectos negativos em detrimento aos positivos. Primeiro, existe uma tendência para o estudo dos fatores que afligem a humanidade, e a expressão e experiência de emoções negativas são responsáveis pela maioria desses conflitos. Em contraste, experiências que promovem felicidade, muitas vezes, passam desapercebidas. Seligman (2002) salienta a II Guerra Mundial como um marco importante para o estudo focado somente nas patologias. Antes desse acontecimento, a Psicologia possuía três missões: curar as doenças mentais; tornar a vida das pessoas mais produtiva e feliz; e, identificar e criar talentos. No entanto, após a guerra, as duas últimas missões foram esquecidas. A necessidade de tratar os veteranos de Guerra e a fundação do Instituto Nacional de Saúde Mental nos Estados Unidos propiciaram vantagens econômicas, profissionais e sociais aos psicólogos e pesquisadores. Dessa forma, as pesquisas e o atendimento clínico concentraram-se no "reparo" dos danos e prejuízos provocados pelas patologias, de acordo com um modelo de doença do funcionamento humano. Esse movimento trouxe benefícios importantes para o fortalecimento e o aperfeiçoamento das terapias e tratamentos para as doenças mentais, mas ao mesmo tempo, enfraqueceu as investigações sobre os aspectos virtuosos dos seres humanos.

Como outros psicólogos, Seligman devotou sua carreira ao estudo das doenças mentais, especialmente da depressão. Ao assumir a presidência da APA, enfatizou os avanços que a Ciência Psicológica havia obtido nos últimos anos; ele salientou que não havia tratamentos disponíveis para nenhuma doença mental no ano de 1947 e que, nos anos 90, 14 diferentes doenças já podiam ser tratadas através de psicoterapia e psicofarmacologia, ou ambas, o que era uma notável vantagem trazida pelo estudo das patologias. No entanto, a Psicologia ainda não era capaz de oferecer ferramentas para ajudar os indivíduos a prosperarem e a florescerem. Segundo Seligman (2002), a Psicologia deveria possibilitar muito mais do que apenas reparar o que está errado, devendo identificar e fortalecer o que está bom. A partir desse desequilíbrio, Seligman juntamente com Csikszentmihalyi iniciam o movimento da Psicologia Positiva.

Diversos pesquisadores têm discutido a origem e o movimento da Psicologia Positiva. Gable e Haidt (2005) abordam os aspectos teóricos, filosóficos e históricos que propiciaram a visão negativa da natureza humana e oferecem três razões que justificariam o interesse dos pesquisadores pela investigação das "fraquezas" dos seres humanos. Primeiramente, apontam a compaixão ou a necessidade de ajudar outras pessoas quando essas estão sofrendo; a segunda razão refere-se à II Guerra Mundial, e a todos os aspectos históricos e pragmáticos que permearam esse acontecimento; e, por último, indicam as próprias teorias sobre os processos psicológicos, que focalizam os eventos negativos. Os autores argumentam que esses fatores deram suporte ao desequilíbrio atual nas investigações científicas, embora existam poucas justificativas empíricas para a visão predominantemente negativa da natureza humana. Por outro lado, afirmam que o movimento da Psicologia Positiva colabora com a evolução da ciência, sendo que em ape- 
nas cinco anos, ele cresceu rápido e começa a explorar novas fronteiras, levando a que o reconhecimento das virtudes humanas possa ajudar a prevenir ou a diminuir os prejuízos causados pelas patologias, pelo estresse e pelas doenças.

Assinala-se que a Psicologia Positiva pretende contribuir para o florescimento e o funcionamento saudável das pessoas, grupos e instituições, preocupando-se em fortalecer competências ao invés de corrigir deficiências. Sheldon e King (2001) a definem como o estudo científico dos aspectos virtuosos usuais presentes nos indivíduos, o que demonstra a preocupação central desse movimento, que seria estudar o que é típico, ordinário e usual na maioria dos indivíduos. Portanto, compreendem os aspectos típicos como sendo os positivos. Esse movimento não implica em condenar o "resto" da Psicologia como negativo; ao contrário, seu objetivo não está em negar o que é ruim, o que vai mal, ou o que é desagradável na vida dos seres humanos, porque reconhece a existência do sofrimento humano, situações de risco e as patologias, entretanto pretende investigar a outra face dessas questões como, por exemplo, a felicidade e o altruísmo. Essa mudança de abordagem oferece novas possibilidades de resposta às antigas questões.

Seligman (2002) afirma que é chegado o momento para a Psicologia Positiva. Sua principal preocupação é ampliar o campo e modificar o foco dos estudos, ou seja, a Psicologia não estar restrita apenas a reparar o que está errado ou ruim, mas (re)construir qualidades positivas; ele afirma que o tratamento psicológico e as pesquisas não devem pretender apenas consertar ou descobrir o que está "quebrado" ou não funciona, mas fomentar e nutrir o que existe de melhor nos indivíduos. Para o autor a Psicologia não é apenas uma filial da Medicina, preocupada com a doença e a saúde, mas é muito mais do que isso, envolvendo também o trabalho, a educação, $\mathrm{o}$ afeto, a superação e o crescimento, fatores que estão intimamente ligados às possibilidades cotidianas e comuns que passam desapercebidas diante das investigações empíricas. Estudar o comum e o positivo pode propiciar o entendimento do que adoeceu ou não vai bem. Porque, caso contrário, parece que a Psicologia se "esquece" de sua premissa básica, uma vez que a atividade profissional indica ou deveria in- dicar essa consciência dos aspectos positivos e ordinários no desenvolvimento dos seres humanos, porque a própria atuação dos psicólogos traz a crença nas habilidades e fatores saudáveis das pessoas, pois, para que existiriam tratamentos se eles não acreditassem na possibilidade de recuperação da boa qualidade de vida? Assim, afirma-se que essa mudança de perspectiva não é exclusiva da Psicologia Positiva, bem como não se trata de algo novo ou diferente na Ciência Psicológica, mas que resgata aspectos que, até então, eram considerados secundários.

\section{O movimento científico da Psicologia Positiva}

O campo da Psicologia Positiva tem oferecido espaço para a investigação empírica dos aspectos virtuosos a partir de métodos científicos rigorosos. Seligman (2003) identifica três importantes pilares para a investigação nessa perspectiva: 1) a experiência subjetiva; 2) as características individuais - forças pessoais e virtudes; 3 ) as instituições e comunidades.

A experiência subjetiva refere-se aos estudos sobre o bem-estar subjetivo, experiências positivas ocorridas no passado (Diener, 2000), emoções positivas (Frederickson, 2002b); e, no presente, a aspectos como felicidade (Myers, 2000; Seligman \& Csikszentmihalyi, 2000) e transcendência - flow (Nakamura \& Csikszentmihalyi, 2002); assim como, no futuro, às relacionadas à esperança (Snyder, Rand \& Sigmon, 2002) e ao otimismo (Carver \& Scheier, 2002).

Em relação às características individuais, são focalizados os estudos relacionados às capacidades para o afeto (Hendrick \& Hendrick, 2002), o perdão (McCullough \& Witvliet, 2002), a espiritualidade (Pargament \& Mahoney, 2002), o talento e a sabedoria (Baltes, Gluck \& Kunzmann, 2002).

E, no nível relacionado ao funcionamento dos grupos, é incentivado o estudo sobre as virtudes cívicas e instituições que possibilitam mudanças dos indivíduos como melhores cidadãos, com o foco direcionado para a responsabilidade, o altruísmo, a tolerância (Turner, Barling \& Zacharatos, 2002) e a ética no trabalho (Handelsman, Knapp \& Gottlieb, 2002).

Investigar esses fatores pode ser eficaz na prevenção de problemas relacionados ao comportamento humano. Nos últimos 50 anos, muitos foram os estudos e pesquisas sobre as diferentes patologias e 
modelos de doença. Entretanto, pouco se conhece sobre prevenção, o que se confirma pela escassez de trabalhos sobre os aspectos saudáveis, mantidos durante o desenvolvimento da doença. Com certeza, o principal progresso dado à prevenção tem ocorrido a partir da construção de competências. Pesquisadores reconhecem que as virtudes e as forças pessoais atuam como agentes protetores e preventivos nas doenças mentais. Conhecer os danos e as fraquezas pessoais não se mostra suficiente para promover a prevenção. É necessário fortalecer as pesquisas e o trabalho clínico sobre as habilidades e as capacidades dos seres humanos. Um dos principais aportes teóricos que oferece espaço para o estudo dessas habilidades refere-se à resiliência. Ryff e Singer (2003) afirmam que o funcionamento positivo humano é mais evidente em contextos de mudanças significativas repletas de situações de risco e de adversidades. A análise da resiliência favorece a compreensão das forças humanas, ou seja, quando ela se expressa, as virtudes e as forças pessoais tornam-se conhecidas e, essa possibilidade produz efeitos importantes na vida dos indivíduos, uma vez que favorece suas potencialidades, tornando-os mais fortes e produtivos.

Masten (2001) reconhece que a resiliência é um fenômeno comum e presente no desenvolvimento de qualquer ser humano. No entanto, geralmente, essas forças são conhecidas pela própria pessoa apenas nos momentos adversos. Para compreender a resiliência, segundo Rutter (1993), é preciso saber a dinâmica das características protetoras, ou seja, como se desenvolveu e modificou a trajetória do indivíduo. Nesse sentido, pode-se perceber a importância do reconhecimento das habilidades e capacidades individuais. E Seligman (2003) salienta que para alcançar sucesso em prevenção é necessário e emergente o investimento no conhecimento científico sobre as virtudes e forças pessoais, o que pode ser uma contribuição da Psicologia Positiva.

O movimento da Psicologia Positiva tem produzido importantes aplicações e avanços científicos. Segundo Snyder e Lopez (2002), um dos passos mais importantes nessa promoção foi a independência do modelo tradicional patológico. Certamente, essa afirmação demonstra uma preocupação de renomados cientistas pelo outro lado - o lado bom dos seres humanos, embora o "lado escuro" ainda atraia atenção de muitos. A Psicologia Positiva não pretende travar batalhas a fim de descobrir ou demonstrar a superioridade de um ou outro modelo explicativo do comportamento humano, mas levar a que se reconheça uma nova abordagem constituída de rigorosos métodos da ciência para a investigação dos fatores que dão significado ao que há de sadio no ser humano; ela pode e

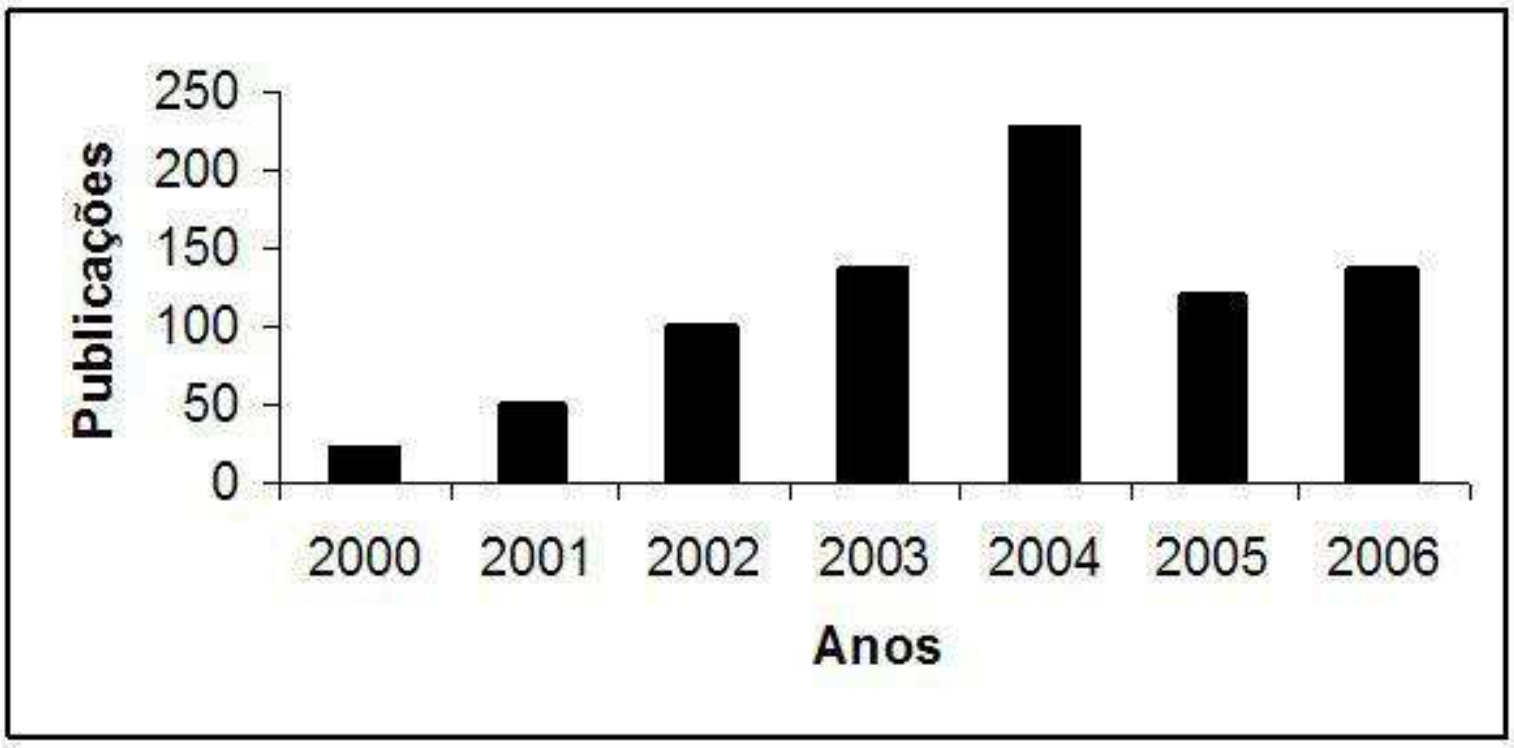

Figura 1. Publicações de Psicologia Positiva no periodo de 2000 a 2006 (Fonte: PsycInfo) 
deve se ocupar de todos os passos metodológicos da Ciência "tradicional" para promover o conhecimento. Muitos pesquisadores têm feito uso dessas premissas para investigar os aspectos virtuosos e as forças existentes nos indivíduos, comunidades e instituições, o que pode ser confirmado através do que mostra o banco de dados do PsychInfo, onde são encontradas 793 referências sobre Psicologia Positiva no período compreendido entre o ano 2000 até 2006 (ver Figura 1).

Constata-se a existência de uma tendência positiva devido ao crescente número de publicações no cenário científico internacional, o que evidencia o interesse dos pesquisadores nessas temáticas. É inegável o crescimento da área, apesar da visível queda no ano de 2005. Além disso, essa ampla gama de estudos demonstra a capacidade e a emergência desse movimento em pouco tempo.

\section{Contribuições da Psicologia Positiva}

Entre as principais contribuições destacam-se a construção de instrumentos de avaliação, modelos de intervenção e aplicação no curso desenvolvimental (Seligman, 2002). Trata-se de uma proposta teórica que pretende criar métodos preventivos através do conhecimento dos fatores protetivos, aprimorar técnicas de avaliação psicológica para identificação das virtudes e dos aspectos positivos e ampliar o escopo de estudo das Ciências Sociais e Humanas. Assim, esforços não têm sido medidos para a criação e aperfeiçoamento de técnicas e instrumentos de medidas a fim de facilitar e promover o desenvolvimento dessa nova área da ciência. A principal missão, no momento, tem sido a operacionalização de instrumentos para a avaliação e a classificação das virtudes e das forças pessoais.

Essa proposta surge em contraponto ao Manual de Estatística e Diagnóstico das Desordens Mentais (DSM), ao possibilitar a identificação dos aspectos saudáveis, mesmo avaliando as patologias. Certamente, o DSM-IV R (American Psychiatric Association, 2000) tem ajudado os profissionais não só a identificarem e diagnosticarem as doenças mentais como, também, a viabilizarem tratamentos para as desordens. No entanto, conforme apontam Wright e Lopez (2002), a partir da discussão do modelo da doença, há um erro comum na prática profissional dos psicólogos: o diagnóstico, o tratamento e as decisões políticas baseiam-se apenas nas deficiências apresentadas pelos pacientes/clientes, ao invés de serem consideradas as forças e as virtudes pessoais do indivíduo e seu ambiente, o que indica que o foco está nos pontos negativos, embora como profissionais da saúde, tenham o conhecimento de que as pessoas exibem uma ampla variedade de comportamentos e capacidades saudáveis; no entanto, na sua atuação cotidiana os aspectos doentes ainda são priorizados. Dessa forma, pesquisas têm sido realizadas a fim de promover a construção de instrumentos para identificação, avaliação e classificação do positivo dos indivíduos, dos grupos e instituições. A Psicologia Positiva tem como principal ambição difundir esse modelo, no mesmo nível exibido pelo da doença, indicado pelo reconhecimento do DSM.

Recentemente, Peterson e Seligman (2004) desenvolveram um sistema de classificação para os aspectos positivos, enfatizando as forças e o caráter denominado Values in Action (VIA) - Classification of Strengths and Virtues Manual. Os autores afirmaram que as forças poderiam ser amplamente classificadas a partir de suas características emocionais, cognitivas, relacionais e cívicas, através de seis grupos de virtudes: sabedoria, coragem, humanidade, justiça, temperamento e transcendência. Essa classificação tem sido considerada universal, uma vez que a avaliação envolveu diferentes culturas, contextos e tempos históricos. O objetivo desse material é prover definições, medidas e intervenções para cada uma das forças de caráter, algumas já publicadas como o livro, Positive Psychology Assesment: A Handbook of Models and Measures (Lopez \& Snyder, 2003). Pesquisadores e terapeutas podem utilizar esses instrumentos para desenvolverem intervenções e ajudarem as pessoas a acentuarem suas possibilidades de florescimento.

A terapia positiva é outra importante contribuição do movimento. Essa modalidade de tratamento visa fortalecer os aspectos saudáveis e positivos dos indivíduos, (re)construir as virtudes e forças pessoais, e ajudar os clientes a encontrarem recursos inexplorados para mudança positiva. Seligman (1995) investigou a psicoterapia e notificou que $90 \%$ dos cli- 
entes sentem-se beneficiados com o tratamento. Destacou, ainda, que esses benefícios não são resultados de uma forma ou modelo particular de psicoterapia ou da eliminação de sintomas, mas, argumentou que uma boa terapia aumenta as forças pessoais já existentes nos clientes, ou seja, modificar somente o que está ruim ou errado é apenas uma parte do trabalho da psicoterapia, sendo preciso também reconstruir e fortalecer o que está bom. A orientação positiva da terapia reconhece que os traços positivos e os comportamentos adaptativos servem como fatores protetores contra os estressores e as dificuldades futuras. Dessa forma, ao tomar conhecimento dos aspectos positivos, as pessoas possuem melhor capacidade para lidar com eventos difíceis, tornando-se, assim, agentes ativos na superação da vulnerabilidade e do risco. Idéias semelhantes foram abordadas no início do século XX por Adler, Jung, Rogers e outros teóricos humanistas, mas foram suprimidas pela principal corrente psicológica da época que enfatizava a patologia.

Atualmente, novas perspectivas teóricas e aplicadas na clínica e no aconselhamento estão em concordância com a proposta da psicoterapia da Psicologia Positiva - o foco nas virtudes e forças pessoais, as capacidades para resolução de problemas e a demonstração de competência. Pesechkian (1997) desenvolveu um sistema de terapia denominado Psicoterapia Positiva baseada na hipótese de que todas as pessoas possuem capacidades para lidar com problemas, o que facilita o reconhecimento e o fortalecimento delas. Robitschek (1998), Robitschek e Cook (1999) propuseram uma orientação que facilita a identificação dos aspectos positivos na psicoterapia, denominada Personal Growth Initiative. Essa é definida como o envolvimento ativo e intencional na modificação e no desenvolvimento como pessoa. Outros aportes teóricos e empíricos têm sido explorados para incrementar a terapia de orientação positiva como, por exemplo, a Hope Therapy (Lopez, Floyd, Ulven \& Snyder, 2000).

A Psicologia Positiva tem, pois, produzido muitos avanços na intervenção psicoterapêutica através da construção de instrumentos psicológicos e novas metodologias de acesso aos seres humanos (Snyder
\& Lopez, 2002). Os estudos enfatizam a prevenção, a identificação e o fortalecimento dos aspectos saudáveis presentes, uma vez que esses agem como fatores de proteção. Nesse sentido, as investigações oferecem técnicas e treinam os profissionais e os clientes para construirem e solidificarem as forças e as virtudes pessoais sistematicamente, numa proposta que pretende modificar o papel de passividade do terapeuta e do cliente, existente no modelo tradicional da doença, e torná-los ativos no processo terapêutico.

Outra importante contribuição da Psicologia Positiva envolve a possibilidade de abordar as questões envolvidas no desenvolvimento das pessoas, reconhecendo que elas e as experiências estão inseridas em contextos sociais e culturais. Certamente, esse movimento não é o único que distingue a importância do ambiente social para o comportamento humano, no entanto, produz uma mudança na teoria psicológica ao conceitualizá-lo como um organismo integrado. Por isso, dedica-se, também, ao estudo do funcionamento de grupos e instituições, por entender que esses ambientes são significativos na vida das pessoas.

Importantes contribuições têm sido feitas por Myers (2000), que descreve como as relações sociais favorecem a felicidade na vida dos indivíduos; Larson (2000) enfatiza as atividades voluntárias para o desenvolvimento das pessoas e Winner (2000) indica o efeito positivo das famílias sobre o florescimento de talentos. Além desses, outros trabalhos têm enfatizado as relações saudáveis no ambiente de trabalho (Turner, Barling \& Zacharatos, 2002) que previnem os conhecidos riscos nele presentes, tais como o estresse e a depressão e, ao mesmo tempo, promovem o bem-estar psicológico e físico. De fato, o foco nos aspectos positivos do trabalho humano ainda é escasso na literatura, o que é corroborado pela própria história da investigação científica em psicologia.

\section{Psicologia Positiva: influências da literatura internacional e avanço no Brasil}

No Brasil, o movimento da Psicologia Positiva ainda não recebeu a devida atenção. Não são encontrados estudos científicos ou publicações nos bancos de dados. Uma busca no Index Psi-Periódicos (www.bvs-psi.org.br) aponta apenas uma referência 
a partir do descritor: Psicologia Positiva. O artigo elaborado por Yunes (2003) introduz a discussão do fenômeno da resiliência vinculado ao movimento da Psicologia Positiva, uma vez que salienta um importante aspecto saudável e positivo do desenvolvimento humano - a superação das adversidades. O conceito de resiliência surgiu há pouco mais de vinte anos na comunidade acadêmica (Masten \& Garmezy, 1985; Rutter, 1981, 1985, 1987, 1993, 1996, 1999).

O primeiro manuscrito sobre resiliência no Brasil foi publicado nas coletâneas da Associação Nacional de Pós-graduação e Pesquisa em Psicologia (Hutz, Koller, \& Bandeira, 1996). No entanto, estes autores não a relacionavam diretamente com a Psicologia Positiva. Só recentemente, foi incorporado ao movimento devido ao seu enfoque nas forças pessoais, que Yunes adotou de Masten (2001) que ofereceu uma importante contribuição ao argumentar que a resiliência é um processo normativo da adaptação, presente na espécie humana e aplicável ao desenvolvimento em ambientes favoráveis ou adversos, afirmando que todos os seres vivos possuem "capacidade" para o desenvolvimento saudável e positivo, o que antes parecia ser exclusivo de alguns indivíduos. Essa afirmação relaciona-se aos temas centrais da Psicologia Positiva. De acordo com Yunes (2003), a Psicologia no Brasil também negligenciou o fenômeno humano da resiliência, essencial para a adaptação e o desenvolvimento durante os períodos de risco, problemas, patologias e tratamento e ela ressalta que a resiliência não emerge de qualidades raras ou especiais, mas que surge de fatos cotidianos e usuais presentes na trajetória e nas relações das crianças, famílias, e comunidades. Masten (2001), também, revela uma perspectiva mais otimista e lança o desafio da compreensão dos processos que podem favorecer ou diminuir o fenômeno da resiliência.

O interesse pelos fenômenos positivos e aspectos saudáveis têm aumentado nesses últimos anos, no Brasil e no mundo; e trata-se de uma mudança de olhar com relação ao humano. Essa nova lente acompanha os preceitos indicados pelo movimento da Psicologia Positiva, mas ainda são escassas as informações sobre essa mudança expressiva que ocorre na Psicologia, tendo-se uma modificação gradual dos estudos brasileiros no seu enfoque e abordagem sobre o desenvolvimento humano.
Uma estratégia teórico-metodológica que tem orientado as pesquisas sobre os aspectos saudáveis das pessoas é a Abordagem Ecológica do Desenvolvimento Humano, proposta por Bronfenbrenner (1979/ 1996, 1986, 1995a, 1995b), que foi reformulada mais recentemente por Bronfenbrenner e Morris (1998) e denominado Bioecológica. Esse modelo propõe uma visão mais ampla do desenvolvimento humano através da interação de quatro núcleos inter-relacionados: o processo, a pessoa, o contexto e o tempo. Dessa forma, possibilita que a atenção investigativa seja dirigida não só para a pessoa e os ambientes imediatos nos quais se encontra, mas também para considerar suas interações e transações com os ambientes mais distantes, dos quais muitas vezes sequer participa diretamente.

Neste modelo, o processo é destacado como o mecanismo responsável pelo desenvolvimento, que é visto através de interação recíproca progressivamente mais complexa de um ser humano ativo, biopsicologicamente em evolução, com as pessoas, objetos e símbolos presentes no seu ambiente imediato (Bronfenbrenner \& Morris, 1998). Estas formas de interação no ambiente imediato são denominadas processos proximais. O segundo componente é a pessoa, que é analisada através de suas características determinadas biopsicologicamente e aquelas construídas na sua interação com o ambiente. $\mathrm{O}$ terceiro refere-se ao contexto, é analisado através da interação de quatro níveis ambientais, denominados como microssistema, mesossistema, exossistema e macrossistema. Finalmente, o quarto componente, o tempo, permite examinar a influência para o desenvolvimento humano de mudanças e continuidades que ocorrem ao longo do ciclo de vida (Bronfenbrenner, 1986), sendo analisado em três níveis no modelo bioecológico: microtempo, mesotempo e macrotempo. Diversas publicações brasileiras têm privilegiado a utilização e a aplicação do modelo bioecológico nos seus estudos (Koller, 2004).

Por fim, a Abordagem Bioecológica do Desenvolvimento Humano e a Psicologia Positiva trazem à tona importantes implicações teóricas, empíricas e práticas que podem e devem ser incorporadas à atuação dos diferentes profissionais e às investigações. Ambas propostas possibilitam a crença no potencial 
do ser humano como um ser ativo capaz de desenvolver estratégias e habilidades para conseguir atingir o desenvolvimento saudável, permitindo um novo olhar para as antigas questões presentes na ciência.

Nesse sentido, a comunidade acadêmica brasileira está despertando para acompanhar os relevantes avanços nessa área. O movimento vem favorecendo uma rápida e complexa produção científica no mundo, rompendo com os ideais epistemológicos vigentes até então. O futuro da Psicologia Positiva pretende "revolucionar" a visão de ser humano, com a construção de uma ciência que focalize os aspectos positivos e salutogênicos dos indivíduos, instituições e grupos, o que parece ser imprescíndivel, numa idéia que não é nova na Psicologia, mas nesse momento favorável ao surgimento e estabelecimento dessa área da ciência.

Um elemento essencial que diferencia esse movimento das tentativas anteriores, realizadas por Rogers e Maslow, é o investimento teórico, metodológico, aplicado e empírico. Myers (2000) afirma que a Psicologia Positiva apresenta uma metodologia imbricada no inquérito sistemático e cientifico.

A psicologia dos últimos 50 anos, que voltou suas energias ao estudo das patologias gerou muitos benefícios, tais como o desenvolvimento de sofisticados métodos longitudinais e experimentais, essenciais para o entendimento do funcionamento humano; transtornos foram descobertos, diagnosticados e tratados; laboratórios, instrumentos de medidas e avaliação confiáveis foram construídos; doenças consideradas incuráveis ou intratáveis foram expostas a intervenções psicológicas e psicofarmacológicas com sucesso; terapias e tratamentos clínicos foram desenvolvidos e aprimorados. Esses estudos devem continuar contribuindo para o avanço científico da Psicologia. É necessário reunir os mesmos esforços para o estudo do outro lado do funcionamento humano - o positivo. A ciência precisa também, como base na promoção da saúde, aprender como proteger e prevenir. Para esses fins, a Psicologia Positiva pretende trazer a compreensão das virtudes, forças pessoais, habilidades promovidas nos contextos de resiliência, averiguar o papel das experiências positivas, e delinear a função das relações positivas com os outros. Além disso, visa esclarecer como todos esses fatores podem contribuir para a saúde física, o bem-estar subjetivo, o funcionamento dos grupos e o florescimento das instituições.

\section{Referências}

American Psychiatric Association. (2000). Diagnostic and statistical manual of mental disorders (Rev.). Washington DC: Author.

Aspinwall, L., \& Staudinger, U. (2003). A psychology of human strengths: Fundamental questions and future directions for a positive psychology. Washington, DC: American Psychology Association.

Baltes, P., Gluck, J., \& Kunzmann, U. (2002). Wisdom: Its structure and function in regulation successful life span development. In C. R. Snyder, \& S. J. Lopez (Eds.), Handbook of positive psychology (pp. 327-347). New York: Oxford University Press.

Bronfenbrenner, U. (1986). Ecology of the family as a context for human development: Research perspectives. Developmental Psychology, 22(6), 723-742.

Bronfenbrenner, U. (1995a). Bioecological model from a life course perspective: Reflections of a participant observer. In P. M. Moen, G. H. Elder, $\&$ K. Lüscher (Eds.), Examining lives in context: Perspectives on the ecology of human development (pp. 599-618). Washington, DC: American Psychological Association.

Bronfenbrenner, U. (1995b). Developmental ecology through space and time: A future perspective. In P. Moen, G. H. Elder, \& K. Luscher (Eds.), Examining lives in context: Perspectives on the ecology of human development (pp. 619-647). Washington, DC: American Psychological Association.

Bronfenbrenner, U. (1996). A ecologia do desenvolvimento humano: Experimentos naturais $e$ planejados. Porto Alegre: Artes Médicas. (Originalmente publicado em 1979).

Bronfenbrenner, U., \& Morris, P. (1998). The ecology of developmental process. Em W. Damon (Ed.), Handbook of child psychology (pp. 993-1027). New York: Wiley \& Sons.

Carver, C., \& Scheier, M. (2002). Optimism. In C. R. Snyder, \& S. Lopez (Eds.), Handbook of positive psychology (pp. 231-256). New York: Oxford University Press. 
Compton, W. (2005). An introdution to positive psychology. Belmont: Thompson Wadsworth.

Diener, E. (2000). Subjective well-being: The science of happiness, and a proposal for a national index. American Psychologist, 55(1), 34-43.

Frederickson, B. L. (2002). Positive emotions. In C. R. Snyder, \& S. J. Lopez (Eds.), Handbook of positive psychology (pp. 120-134). New York: Oxford University Press.

Gable, S., \& Haidt, J. (2005). Positive psychology. Review of General Psychology, 9, 1089-2680.

Handelsman, M., Knapp, S., \& Gottlieb, M. (2002). Positive ethics. In C. R. Snyder, \& S. J. Lopez (Eds.), Handbook of positive psychology (pp. 731-744). New York: Oxford University Press.

Hendrick, S., \& Hendrick, C. (2002). Love. In C. R. Snyder, \& S. Lopez (Eds.), Handbook of positive psychology (pp. 472-484). New York: Oxford University Press.

Hernandez, J. (2003). Psicologia positiva e psicologia Humanista: Aproximações teóricas e conceituais. Revista de Psicologia da UnC, 1(1), 24-30.

Hutz, C. S., Koller, S. H., \& Bandeira, D. R. (1996). Resiliência e vulnerabilidade em crianças em situação de risco. Coletâneas da ANPEPP, 1(12), 79-86.

Keyes, C. L. M., \& Haidt, J. (2003). Flourishing: Positive psychology and the life well lived. Washington DC: American Psychological Association.

Koller, S. (2004). Ecologia do desenvolvimento humano: Pesquisa e intervenção no Brasil. São Paulo: Casa do Psicológo.

Larson, R. (2000). Toward a psychology of positive youth development. American Psychologist, 55(1), 170-183.

Lopez, S., Floyd, R., Ulven, J., \& Snyder, C. (2000). Hope therapy: Helping clients build a house of hope. In C. R. Snyder (Ed.), Handbook of hope: Theory, measures, and applications (pp. 123166). San Diego: Academic Press.
Lopez, S. J., \& Snyder, C. R. (2003). Positive psychological assessment. Washington, DC: American Psychological Association.

Maslow, A. (1954). Religions, values, and peakexperiences. Columbus: Ohio State University Press.

Masten, A. S. (2001). Ordinary magic: Resilience processes in development. American Psychologist, 56(3), 227-238.

Masten, A. S., \& Garmezy, N. (1985). Risk, vulnerability and protective factors in developmental psychopathology. In B. B. Lahey, \& A. E. Kazdin (Eds.), Advances in clinical child psychology (pp.1-52). New York: Plenum Press.

McCullough, M., \& Witvliet, C. (2002). The psychology of forgiveness. In C. R. Snyder, \& S. J. Lopez (Eds.), Handbook of positive psychology (pp. 446-458). New York: Oxford University Press.

Myers, D. G. (2000). The funds, friends, and faith of happy people. American Psychologist, 55(1), 56-67.

Nakamura, J., \& Csikszentmihalyi, M. (2002). The concept of flow. In C. R. Snyder, \& S. J. Lopez (Eds.), Handbook of positive psychology (pp. 89-105). New York: Oxford University Press.

Pajares, F. (2001). Toward a positive psychology of academic motivation. Journal of Educational Research, 95(1), 27-36.

Pargament, K., \& Mahoney, A. (2002). Spirituality: Discovering and conserving the sacred. In C. R. Snyder, \& S. J. Lopez (Eds.), Handbook of positive psychology (pp. 646-659). New York: Oxford University Press.

Pesechkian, N. (1997). Positive psychoterapy: Theory and practice of a new method. Berlin: Springer-Verlag.

Peterson, C., \& Seligman, M. (2004). Character strengths and virtues: A classification and handbook. Washington, DC: American Psychological Association. 
Resnick, S., Warmoth, A., \& Serlin, I. (2001). The humanistic psychology and positive psychology connection: Implications for psychotherapy. Journal of Humanistic Psychology, 41(1), 73-101.

Robitschek, C. (1998). Personal growth initiative: The construct and its measure. Measurement and Evaluation in Counseling and Development, 30, 183-198.

Robitschek, C., \& Cook, S.W. (1999). The influence of personal growth initiative and coping styles on career exploration and vocational identity. Journal of Vocational Behavior, 54, 127-141.

Rogers, C. R. (1959). A theory of therapy, personality, and interpersonal relationships, as developed in the client-centered framework. In S. Koch (Ed.), Psychology: A study of a science: Formulations of the person and the social context (pp. 184256). New York: McGraw-Hill.

Rutter, M. (1981). Stress, coping and development: Some issues and some questions. Journal of Child Psychology \& Psychiatry, 22(4), 323-356.

Rutter, M. (1985). Resilience in the face of adversity: Protective factors and resistance to psychiatric disorder. British Journal of Psychiatric, 147, 598-611.

Rutter, M. (1987). Psychosocial resilience and protective mechanisms. American Orthopsychiatry Association, 57(3), 316-331.

Rutter, M. (1993). Resilience: Some conceptual considerations. Journal of Adolescent Health, 14, 626-631.

Rutter, M. (1996). Psychosocial resilience and protective mechanisms. Em J. Rolf, A. S. Masten, D. Cicchetti, K. H. Nuechterlein, \& S. Weintraub (Eds.), Risk and protective factors in the development of psychopatology (pp. 181-214). New York: Cambridge University.

Rutter, M. (1999). Resilience concept and findings: implications for family therapy. Journal of Family Therapy, 21, 119-144.
Ryff, C. D., \& Singer, B. (2003). Flourishing under fire: Resilience as a prototype of challenged thriving. In C. L. M. Keyes, \& J. Haidt (Eds.), Flourishing: Positive psychology and the life well-lived (pp. 15-36). Washington, DC: American Psychological Association.

Seligman, M. (1995). The effectiveness of psychotherapy: The consumer reports study. American Psychologist, 50, 965-974.

Seligman, M. (2002). Positive psychology, positive prevention, and positive therapy. In C. R. Snyder, \& S. J. Lopez (Eds.), Handbook of positive psychology (pp. 3-9). New York: Oxford University Press.

Seligman, M. (2003). Foreword: The past and future of positive psychology. In C. L. M. Keyes, \& J. Haidt (Eds.), Flourishing: Positive psychology and the life well lived (pp. 11-20). Washington DC: American Psychological Association.

Seligman, M., \& Csikszentmihalyi, M. (2000). Positive psychology: An introduction. American Psychologist, 55, 5-14.

Sheldon, K. M., \& King, L. (2001). Why positive psychology is necessary? American Psychologist, 56, 216-217.

Schmuck, P., \& Sheldon, K. M. (2001). Life goals and well-being: Towards a positive psychology of human striving. Seattle: Hogrefe \& Huber.

Snyder, C. R., \& Lopez, S. J. (2002). Handbook of positive psychology. New York: Oxford University Press.

Snyder, C. R., Rand, K. L., \& Sigmon, D. R. (2002). Hope theory: A member of the positive psychology family. In C. R Snyder, \& S. J. Lopez, (Eds.), Handbook of positive psychology (pp.257-276). New York: Oxford University Press.

Sollod, R. (2000). Religious experience: Religious and spiritual practices. In Encyclopedia of American Psychology, 7, (pp.52-57). Washington, DC: American Psychological Association.

Taylor, E. (2001). Positive psychology and humanistic psychology: A reply to Seligman. Journal of Humanistic Psychology, 41, 13-29. 
Turner, N., Barling, J., \& Zacharatos, A. (2002). Positive psychology at work. In C.R. Snyder \& S.J. Lopez (Eds.), Handbook of positive psychology (pp 715-728). New York: Oxford University Press.

Yunes, M. A. (2003). Psicologia positiva e resilência: O foco no indivíduo e na família. Psicologia em Estudo, 8(especial), 75-84.

Winner, E. (2000). The origins and ends of giftedness. American Psychologist 55, 159-169.

Wright, B., \& Lopez, S. (2002). Widening the diagnostic focus: A case for including human strengths and environmental resources. In C.R. Snyder, \& S.J. Lopez (Eds.), Handbook of positive psychology (pp. 26-44). New York: Oxford University Press.

Endereço para correspondência:

Simone dos Santos Paludo. UFRGS, Instituto de Psicologia, Rua Ramiro Barcelos 2600/104, CEP 90035-003, Porto Alegre - RS, Brasil. E-mail: cep_rua@ufrgs.br

Artigo recebido em 09/01/2007.

Aceito para publicação em 30/05/2007.

\section{Apoio CAPES}

Este estudo faz parte das atividades de Doutorado em Psicologia (UFRGS) da primeira autora, orientada pela segunda.

Simone Paludo é Psicóloga, Doutoranda e Mestre em Psicologia do Desenvolvimento do Curso de Pós-Graduação em Psicologia da Universidade Federal do Rio Grande do Sul e Professora da Universidade Federal de Rio Grande (FURG). Membro do Centro de Estudos Psicológicos sobre Meninos e Meninas de Rua (CEP-RUA/UFRGS). Vice-coordenadora do CEP-RUA/FURG.
Sílvia Helena Koller é Psicóloga, Doutora em Educação (PUCRS), Pesquisadora do CNPq e Professora do Curso de Pós-Graduação em Psicologia do Desenvolvimento da Universidade Federal do Rio Grande do Sul. Coordenadora do Centro de Estudos Psicológicos sobre Meninos e Meninas de Rua (CEPRUA/UFRGS). 